\title{
Ten years of positron and electron solar modulation measured by the PAMELA experiment.
}

\author{
Riccardo Munini; $^{* 1}$, V. di Felice ${ }^{2,3}$, M. Boezio ${ }^{1}$, O. Adriani ${ }^{4,5}$, G. C. Barbarino ${ }^{6,7}$, \\ G. A. Bazilevskaya ${ }^{8}$, R. Bellotti ${ }^{9}, 10$, E. A. Bogomolov ${ }^{11}$, M. Bongi $i^{3,5}$, V. Bonvicini ${ }^{1}$, \\ S. Bottai ${ }^{5}$, A. Bruno ${ }^{9,10}$, F. Cafagna ${ }^{10}$, D. Campana ${ }^{7}$, P. Carlson ${ }^{12}$, M. Casolino ${ }^{2,14}$, \\ G. Castellini ${ }^{15}$, C. De Donato ${ }^{2}$, C. De Santis ${ }^{13}$, A. M. Galper ${ }^{16}$, A. V. Karelin ${ }^{16}$, \\ S. V. Koldashov ${ }^{16}$, S. Koldobskiy ${ }^{16}$, S. Y. Krutkov ${ }^{11}$, A. N. Kvashnin ${ }^{8}$, \\ A. Leonov ${ }^{16}$,V. Malakhov ${ }^{16}$, L. Marcelli ${ }^{13}$, M. Martucci ${ }^{13,17}$, A. G. Mayorov ${ }^{16}$, \\ W. Menn ${ }^{18}$, M. Merge ${ }^{2,13}$, V. V. Mikhailov ${ }^{16}$,E. Mocchiutti ${ }^{1}$, A. Monaco ${ }^{9,10}$, N. Mori ${ }^{5}$, \\ G. Osteria ${ }^{7}$, B. Panico ${ }^{7}$ P. Papini ${ }^{5}$, M. Pearce ${ }^{12}$, P. Picozza ${ }^{2,13}$, M. Ricci ${ }^{17}$, \\ S. B. Ricciarini ${ }^{15}$, M. Simon ${ }^{18}$, R. Sparvoli ${ }^{2,13}$, P. Spillantini ${ }^{4,5}$, Y. I. Stozhkov ${ }^{8}$, \\ A. Vacchi ${ }^{1,19}$, E. Vannuccini ${ }^{5}$, G. Vasilyev ${ }^{11}$, S. A. Voronov ${ }^{16}$, Y. T. Yurkin ${ }^{16}$, \\ G. Zampa ${ }^{1}$, N. Zampa ${ }^{1}$, M. S. Potgieter ${ }^{20}$, J.L. Raath ${ }^{20}$, \\ ${ }^{1}$ INFN, Sezione di Trieste I-34149 Trieste, Italy \\ ${ }^{2}$ INFN, Sezione di Rome "Tor Vergata”, I-00133 Rome, Italy \\ ${ }^{3}$ Agenzia Spaziale Italiana (ASI) Science Data Center, I-00044 Frascati, Italy \\ ${ }^{4}$ University of Florence, Department of Physics, I-50019 Sesto Fiorentino, Florence, Italy \\ ${ }^{5}$ INFN, Sezione di Florence, I-50019 Sesto Fiorentino, Florence, Italy \\ ${ }^{6}$ University of Naples "Federico II", Department of Physics, I-80126 Naples, Italy \\ ${ }^{7}$ INFN, Sezione di Naples, I-80126 Naples, Italy \\ ${ }^{8}$ Lebedev Physical Institute, RU-119991, Moscow, Russia \\ ${ }^{9}$ University of Bari, Department of Physics, I-70126 Bari, Italy \\ ${ }^{10}$ INFN, Sezione di Bari, I-70126 Bari, Italy \\ ${ }^{11}$ Ioffe Physical Technical Institute, RU-194021 St. Petersburg, Russia \\ ${ }^{12}$ KTH, Department of Physics, and the Oskar Klein Centre for Cosmoparticle Physics, AlbaNova \\ University Centre, SE-10691 Stockholm, Sweden \\ ${ }^{13}$ University of Rome "Tor Vergata”, Department of Physics, I-00133 Rome, Italy \\ ${ }^{14}$ RIKEN, Advanced Science Institute, Wako-shi, Saitama, Japan \\ 15 IFAC, I-50019 Sesto Fiorentino, Florence, Italy \\ ${ }^{16}$ National Research Nuclear University MEPhI, RU-115409 Moscow \\ 17 INFN, Laboratori Nazionali di Frascati, Via Enrico Fermi 40, I-00044 Frascati, Italy \\ 18 Universität Siegen, Department of Physics, D-57068 Siegen, Germany \\ ${ }^{19}$ University of Udine, Department of Mathematics and Informatics, I-33100 Udine, Italy \\ ${ }^{20}$ North-West University, Centre for Space Research,2520 Potchefstroom, South Africa
}


The satellite-borne PAMELA experiment was launched on the 15th June 2006 from the Baikonur cosmodrome. Till January 2016 PAMELA has detected the charged component of cosmic-rays (CRs) over a wide energy range. Due to its long flight duration PAMELA represents an ideal detector for cosmic-ray solar modulation studies. Moreover, the capability to measure particles of the same mass with opposite charge allows to investigate the charge-sign dependent solar modulation. The results on the positron and electron intensity variation at Earth over the 23rd solar minimum (July 2006 - January 2009) till the middle of the 24rd solar maximum (December 2015), will be presented. The positron to electron ratio shows a clear time variation interpreted as solar modulation sign-charge dependence introduced by particle drifts. The effect of the polarity reversal of the heliospheric magnetic field, which took place between 2013 and 2014, is also distinctly visible from the PAMELA data. These results provide the first clear indication of how drift effects unfold with time during different phases of the solar activity and their dependence on the particle rigidity and the cyclic polarity of the solar magnetic field.

35th International Cosmic Ray Conference - ICRC2017

10-20 July, 2017

Bexco, Busan, Korea

${ }^{*}$ Speaker. 


\section{Introduction}

Electrons and positrons are a natural component of the cosmic radiation and are both produced in the interactions between cosmic-ray nuclei and the interstellar matter. Additionally, since the observed electron flux is about an order of magnitude larger than the positron one (e.g. [1]), most of the electrons must be of primary origin, probably accelerated to high energy by astrophysical shocks generated at sites like supernova remnants (e.g. [2]).

After having propagate through the Galaxy, cosmic ray electrons and positrons traverse the heliosphere, where they are scattered by the irregularities of the turbulent HMF embedded into the solar wind and undergo convection and adiabatic deceleration in the expanding solar wind. As a consequence, the intensity of CRs at Earth decreases with respect to the local interstellar spectrum [3]. Solar modulation has large effects on low energy CRs (less than a few $\mathrm{GeV}$ ) and has negligible effects above energies of a few tens of GeV. Moreover, due to the 11-year solar activity cycle, the intensity of CRs inside the heliosphere changes with time. During solar minimum periods, the intensity of CRs is higher with respect to periods of solar maximum. This behavior is well shown in Figure 1 where the Oulu count rate normalized to 2006 is presented.

On top of the time dependence, a charge sign dependence of the solar modulation is expected. The gradients and curvatures present in the HMF induce drift motions that depend on the particle charge sign. During so-called A $<0{ }^{1}$ polarity cycles such as solar cycle 23 , when the heliospheric magnetic field is directed toward the Sun in the northern hemisphere, negatively charge particles undergo drift motion mainly from the polar to the equatorial regions and outwards along the heliospheric current sheet. Positively charged particles drift mainly in opposite directions. The situation reverses when the solar magnetic field changes its polarity at each solar maximum. The charge-sign dependence introduces a 22-years cycle also visible in the neutron monitor count rate. As shown in Figure 1, during $A>0$ polarity cycle the shape of neutron monitor count rate during solar minima are flatter with respect to $A<0$ polarity cycle when the peaks are a sharper.

The coincidental study of positively and negatively charged particles allows to understand the contribution of drift motion to the propagation of CRs. Furthermore, extending these measurements to solar maximum conditions and reversal of the magnetic field polarity allows to study how drift effects evolve with solar activity and if they actually account for the differences in the experimental results. The low energies $(<5 \mathrm{GeV})$ time-evolution of electron and positron intensity from mid 2006 to late 2015 through the polarity reversal of the solar magnetic field with PAMELA data will be presented.

\section{The PAMELA instrument}

The PAMELA experiment was launched on June 15th 2006 from the Bajkonur cosmodrome on-board the Resurs DK1 satellite and, since then, it has almost continuously taked data. The instrument followed a high inclination $\left(70^{\circ}\right)$ orbit, ideal condition to observe the solar modulation of galactic cosmic rays down to very low energies $(<70 \mathrm{MeV}$ for electrons and positrons). The weight of PAMELA is $470 \mathrm{~kg}$ for a total power consumption of $355 \mathrm{~W}$.

\footnotetext{
${ }^{1}$ In the complex sun magnetic field the dipole term nearly always dominates the magnetic field of the solar wind. A is defined as the projection of this dipole on the solar rotation axis.
} 


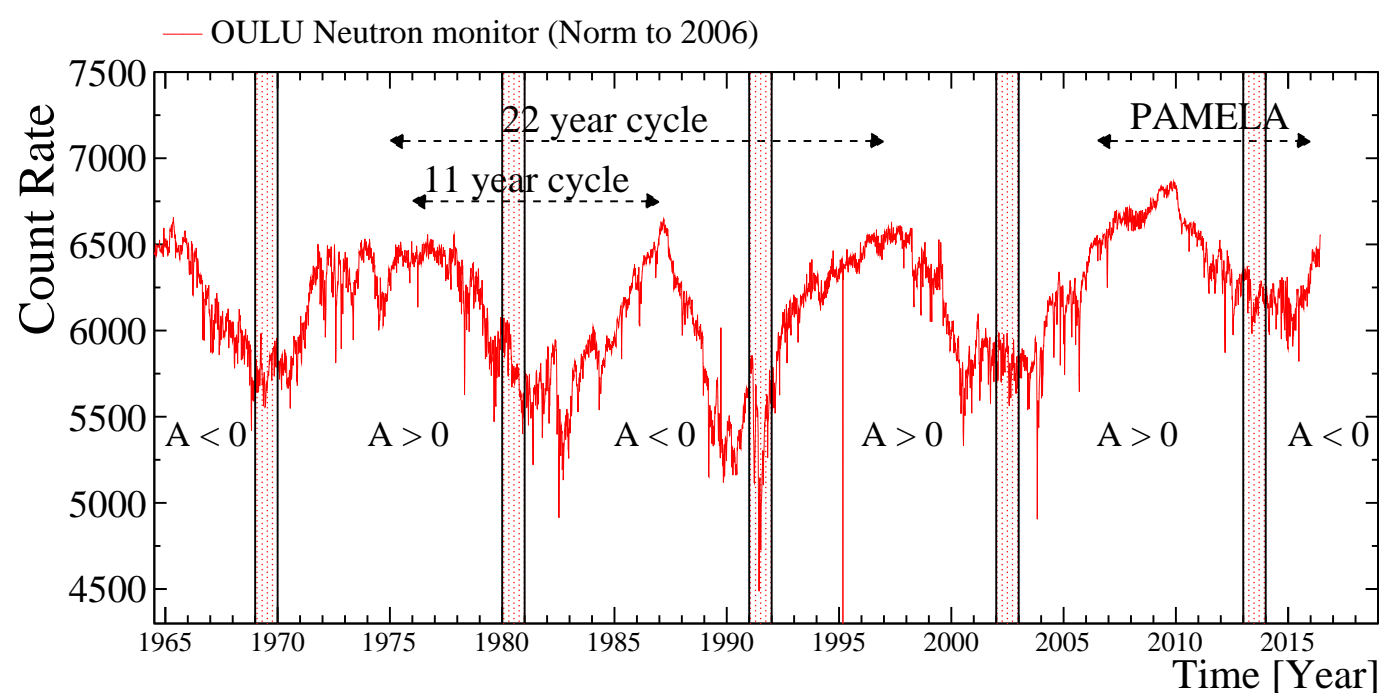

Figure 1: Oulu neutron monitor count rate normalize to 2006. Data taken from from http://cosmicrays.oulu.fi/. Red shaded areas represent the time interval during which the process of polar field reversal took place.

The PAMELA instrument [5] was designed and built to study the antimatter component of cosmic rays from tens of $\mathrm{MeV}$ up to hundreds of $\mathrm{GeV}$ and with a significant increase in statistics with respect to previous experiments. To obtain this goal the apparatus was optimized for the study of $\mathrm{Z}=1$ particles and to reach a high level of positron-proton discrimination.

The apparatus is shown in Figure 2 and comprises the following subdetectors (from top to bottom): a Time-of- Flight (ToF) system, a magnetic spectrometer, an anticoincidence system, an electromag- netic imaging calorimeter, a shower tail catcher scintillator and a neutron detector.

The PAMELA detector is built around a permanent magnet which host the tracking system composed of six planes of double-sided silicon sensors, which form the magnetic spectrometer. The magnetic spectrometer measures the particle rigidity $\mathrm{r}=\mathrm{pc} / \mathrm{Ze}^{2}$ and the ionization energy losses $(\mathrm{dE} / \mathrm{dx})$. The rigidity measurement is done through the reconstruction of the trajectory based on the impact points on the tracking planes and the resulting determination of the curvature due to the Lorentz force. The Time-of-Flight (ToF) system comprises three double layers of plastic scintillator paddles with the first two placed above and the third immediately below the magnetic spectrometer. The ToF system provides the measurements of the particle velocity combining the time of passage information with the track length derived from the magnetic spectrometer. From the information on the particle incoming direction and the curvature in the spectrometer negatively charged particles (electrons) are from positively charged particles (positrons).

The sampling imaging calorimeter has 16.3 radiation lengths (0.6 interaction lengths) and is the main sub-detector used for hadron-lepton separation. The topological and energetic informations of the shower development in the calorimeter allows to reach a rejection power of proton against positron of the order of $10^{-4}$. The shower tail catcher and the neutron detector beneath provide additional information for the discrimination. An anticoincidence system is used to reject

\footnotetext{
${ }^{2} \mathrm{p}$ and $\mathrm{Ze}$ are respectively the particle momentum and charge, and $\mathrm{c}$ the speed of light
} 


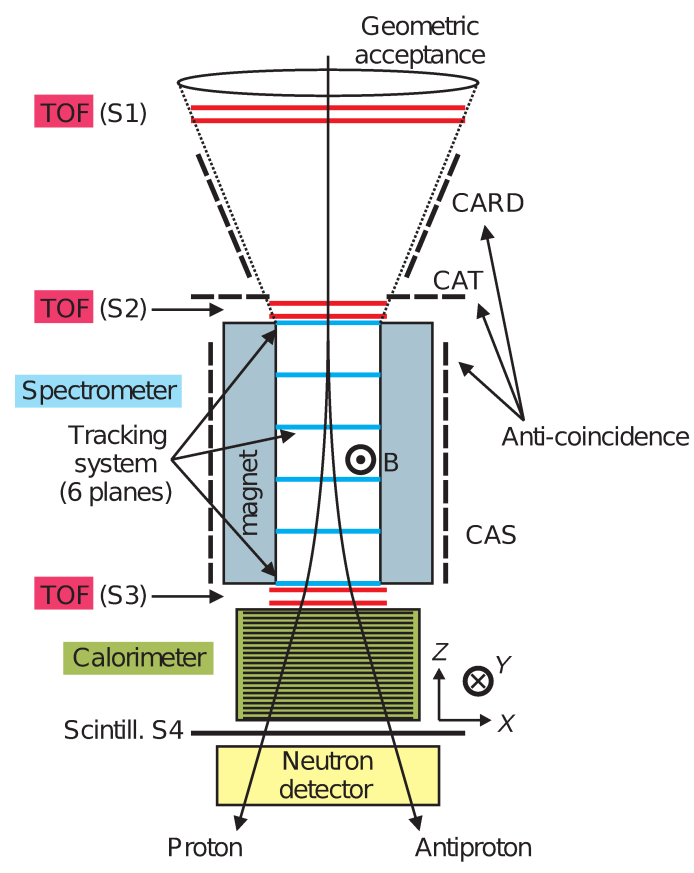

Figure 2: PAMELA and its sub-detectors.

spurious events in the off-line phase.

\section{Analysis and results}

A set of cuts were developed in order to select electrons and positrons from PAMELA data. Events with a single reconstructed track were selected requiring to be inside a fiducial volume bounded $0.15 \mathrm{~cm}$ from the magnet cavity walls in order to avoid interaction with the magnetic walls which could degrade the tracker performance. Particles with $\mathrm{Z}=1$ were selected by means of the ionization losses in the tracker and ToF planes. The ionization energy losses selections also rejects non-relativistic proton, antiprotons and pions ${ }^{3}$ up to 800 and $200 \mathrm{MV}$ respectively. Above this energies hadron contamination was removed with information coming from the electromagnetic calorimeter. Several calorimetric selections based on the topological development of the electromagnetic shower were defined. These selections allowed an almost complete rejection of the proton, antiproton and pion contamination in the rigidity range considered.

Galactic positrons and electrons were selected in three different energy intervals: $0.5-1.0$ $\mathrm{GeV}, 1.0-2.5 \mathrm{GeV}$ and $2.5-5.0 \mathrm{GeV}$. The positron to electron ratio was measured on three-month time periods between July 2006 and December 2015. This energy and time division was chosen as the best balance between the statistics, the energy resolution and the time resolution. Figure 3 shows the results on the time dependence of the positron to electron ratio [6]. Data were normalized to the values measured between July and December 2006. The red shaded area represents the time interval during which the process of polar field reversal took place [7].

\footnotetext{
${ }^{3}$ Both positive and negative pions are produced by the interaction of primary cosmic rays nuclei with the aluminum container that contains the PAMELA instrument.
} 

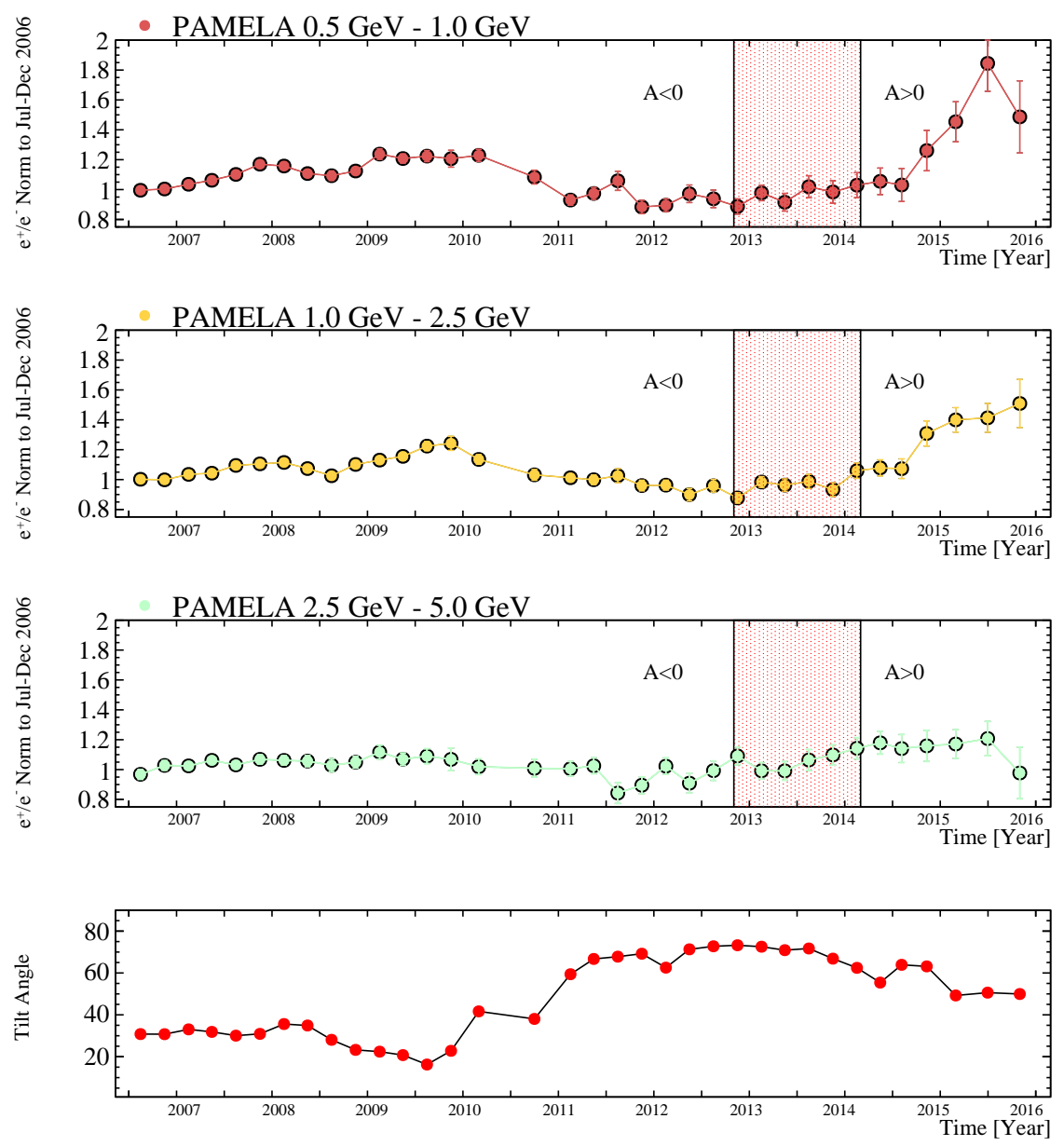

Figure 3: The positron to electron ratios normalized to July - December 2006 measured at Earth by the PAMELA experiment for three different energy intervals. The colored lines provide connection among the points. The shaded area corresponds to the period with no well defined HMF polarity [7]. Bottom panel: the tilt angle as a function of time.

The results show a time dependence of the positron to electron ratio. In the first two energy intervals of Figure $3(0.5-1$ and $1-2.5 \mathrm{GeV})$ an increase of the ratio was observed up to the end of 2009. During this time period positrons at Earth increased about $20 \%$ more than electrons. For the third energy interval $(2.5-5.0 \mathrm{GeV})$ this increase was $\sim 10 \%$.

Until the middle of 2013 the ratio remained constant and slowly increased up to the middle of 2014 when a sudden rise was observed up to late 2015 for the first two panels of Figure 3 where positrons increased respectively about $80 \%$ and $50 \%$ more than electrons. This sudden rise is not observed for the highest energy interval, where the positrons increased only about $20 \%$ more than electrons. The sudden rise measured during this period appears to be a consequence of the polarity reversal of the HMF.

The trends in the observational data shown in Figure 3 can be interpreted in terms of particle 


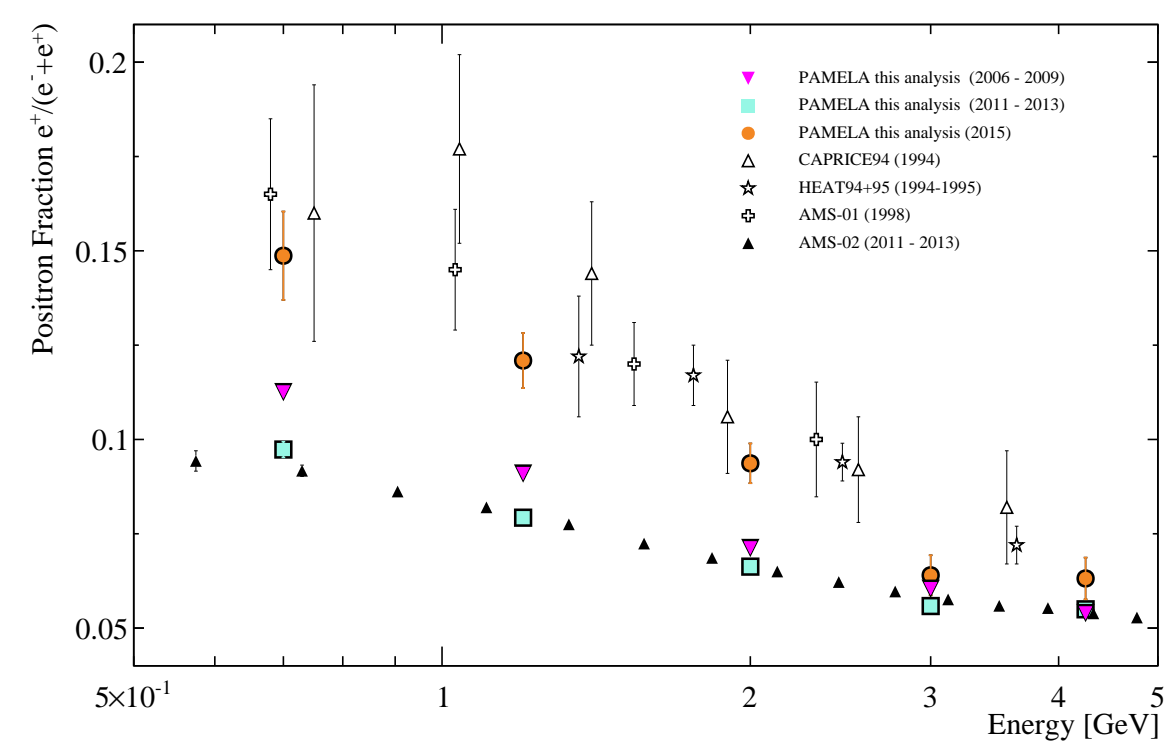

Figure 4: The positron fraction derived in this work for three time periods: July 2006-December 2009, (solar minimum, as in [8]), May 2011-November 2013 (as AMS-02 results [9]), January-December 2015, along with other recent measurements: HEAT94+95 [10], CAPRICE94 [11], AMS-01 [12], AMS-02 [9]. The results from $[10,11,12]$ refer to the previous $\mathrm{A}>0$ solar cycle.

drifts. For the period 2006 to 2009 , the tilt angle ${ }^{4}$ decreased slowly to reach a minimum value at the end of 2009 as shown from bottom panel of Figure 3. During this $A<0$ magnetic polarity cycle, positrons drifted towards the Earth mainly through the equatorial regions of the heliosphere, encountering the changing wavy current sheet, while electrons drifted inwards mainly through the polar regions of the heliosphere and were consequently less influenced by the current sheet. The positron flux therefore increased relatively more than the electron flux with a decreasing tilt angle until the end of 2009. From 2010 onwards, the tilt angle increased sharply so that the positron flux also decreased proportionally faster than the electron flux and the ratio $e^{+} / e^{-}$decreased. This continued until increased solar activity influenced both fluxes equally and the ratio $e^{+} / e^{-}$became steady. From the end of 2012, the solar magnetic field had gone into a reversal phase, which lasted until the beginning of 2014. After this turbulent reversal phase (from $A<0$ to $A>0$ ) the positrons gradually started to drift inwards through the polar regions of the heliosphere to the Earth while the electrons started to drift inwards through the equatorial regions so that the positron flux increased proportionally more than for electrons.

The charge-sign dependence introduced by drift motion is also visible in Figure 4 were the positron fraction measured in this analysis and from other experiments are shown. A good agreement between these data and the AMS-02 results can be noticed. Moreover, the positron fraction measured in 2015 draws near to the measurements [10,11, 12] from the previous $\mathrm{A}>0$ solar cycle

\footnotetext{
${ }^{4}$ In the context of this charge-sign dependent modulation, the tilt angle [?] of the wavy heliospheric current sheet is the most appropriate proxy for solar activity.
} 
in the 90's.

\subsection{Conclusions}

The time evolution of the positron to electron ratio measured with PAMELA data between July 2006 and December 2015 was presented. The positron to electron ratio over time exhibit a clear deviation from one indicating the presence of a charge-sign dependence which affects the propagation of the cosmic rays (below $\sim 5 \mathrm{GV}$ ) in the heliosphere. This charge-sign dependence was interpreted in terms of drift motion. The effects of the polarity reverse of the solar magnetic field was also visible in the positron to electron ratio as a sudden increase after 2014. The positron fraction measured in three different periods of time was also presented. These results show how the positron fraction below $\sim 5 \mathrm{GV}$ is not constant over time due to the the charge sign dependence of the solar modulation.

We acknowledge partial financial support from The Italian Space Agency (ASI) under the program "Programma PAMELA - attivita' scientifica di analisi dati in fase E". We also acknowledge support from Deutsches fur Luft- und Raumfahrt (DLR), The Swedish National Space Board, The Swedish Research Council, The Russian Space Agency (Roscosmos) and Russian Science Foundation. M. Potgieter and J. L. Raath acknowledge the partial financial support from the South African Research Foundation (NRF) under the SA-Italy Bilateral Programme.

\section{References}

[1] J. A. De Shong and R. H. Hildebrand and P. Meyer 1964 Phys. Rev. Lett. 123

[2] G. E. Allen and others 1997 Astrophys. J. Lett. 487 L97

[3] M. S. Potgieter 2013 Living Reviews in Solar Physics 103

[4] X. Sun and J. T. Hoeksema and Y. Liu and J. Zhao 2015 Astrophys. J. 798114

[5] P. Picozza and others 2007 Astropart. Phys. 27296

[6] O. Adriani and others 2016 Phys. Rev. Lett. 116241105

[7] X. Sun and J. Hoeksema J. T. and Liu Y. and Zhao J. 2015 Astrophys. J. 798114

[8] O. Adriani and others 2013 Phys. Rev. Lett. 111081102

[9] L. Accardo and others 2014 Phys. Rev. Lett. 113121101

[10] S. W. Barwick and others 1997 Astrophys. J. 482 L191

[11] M. Boezio and others 2000 Astrophys. J. 532653

[12] J. Alcaraz and others 2000 Phys. Lett. B 48410 